\title{
Reasons for a Careful Design of Fuzzy Sets*
}

\author{
Enric Trillas and Claudio Moraga
}

European Centre for Soft Computing, 33600 Mieres, Asturias, Spain

\begin{abstract}
This paper is, basically, a reflection on modeling, starting by asking what happens when the meaning of a predicate $P$ is interpreted by the membership function of a fuzzy set that, labeled $P$, is designed with the information available to the designer on the use of the predicate in the corresponding universe of discourse. In it, the modification of the meaning that $P$ can suffer by its identification with the membership function of the fuzzy set, is analyzed and discussed. It is argued that what can be done for reaching a least possible modification of the meaning, is just a careful design of the membership function. This reflection is further continued at the level of linguistic variables, fuzzy if-then rules, and rule-based systems. It is concluded that as argued in the case of fuzzy sets, the involved operations in rules and systems should be at least carefully chosen, if not specially designed.
\end{abstract}

Keywords: Models, Quantity-Predicates, Collectives, Elemental Meaning, Fuzzy sets, Ideal and Practical membership functions, Design.

\section{Introduction}

What is a model? A model is the representation of relevant parts of a system, in order to understand the functionality of the (modeled parts of the) system, to be able to possibly control its behavior, and eventually, to predict its performance.

How should a model be developed? A model builder has to consider first, who is going to use the model, and which are his/her level of knowledge and expectations for the model. Then choose a possibly formal frame, where the user can understand the representation (of the relevant parts of the system), the functionality may be simulated, and the expectations may be satisfied. (For instance, if a user is (just) interested in 'controlling' a washing machine to obtain 'good results', a User Handbook would be an appropriate "model": a textual/pictorial model. If the user wants to understand how the machine works, a totally different model would be needed, where the User Handbook might be just a small, almost insignificant, component.)

If the users are Engineers, they will rather be acquainted with hierarchical models. At the highest level of abstraction block diagrams are used,

*This work was partially supported by the Foundation for the Advancement of Soft Computing, Mieres, Asturias, Spain. representing the main components of the system (specified just by names) and their interconnectivity. Blocks are refined to a next lower level of abstraction, where models with possibly different formalisms may be required. The refinements are continued until reaching a functionality level, where mathematical formalisms, quite often systems of differential equations, take over. Refinements end at the hardware physical level. It is interesting to mention that this method of hierarchical modeling has been extended to many other areas. See, e.g. [7] for examples of block diagrams of the brain.

As it is typical in Engineering when for some purpose a physical system has to be represented at the level of functionality in mathematical terms, the first choice is to apply an abstract mathematical formalism, with which the user is familiar. For building a model, it is necessary to know previously on which theory, presuppositions, equations, etc., it can be based in the context where the current problem is inscribed. When all this is done, and provided a general mathematical model is constructed, it should be tuned according to both its own prerequisites, and the data coming from the reality to which it will be applied; that is, the model should be adapted and particularized to the current context the system is inscribed in. On the contrary, the model would not reflect well enough the current system, the outcome solutions will not allow to foresee the real behavior of it, and the representation will be, at least partially, a failure.

For such endeavor, a plan of actuation sometimes can be designed. A plan of design that, when successful, finally ends with the freezing of the model once all in it that is variable, like parameters, initial and border conditions, etc., is contextually fixed [22]. Then, and after some additional checking with the real behavior of the system, the model can be actually applied. This process is sometimes known as the 'design of the system'. Mainly after the appearance of the new area of research called "Computing with Words", efforts have been done, to develop mathematical models for words, as a first step to achieve at some time in the future, computable formal models of language. This, however, should be done within a process of design.

A very simple example of a design process, is given by the case the behavior of the linguistic negation «not», appearing in some expressions going to be represented in fuzzy terms, is contextually analyzed and consequently "decided" that it is not only a strong negation, but can correspond 
with one among those in the parameterized family $N_{p}(x)=(1-x) /(1+p x)$, with $p>-1$, and the parameter $p$ is yet to be fixed by using some more contextual information. If, for instance and for some plausible reason, it were known that $N_{p}(1 / 2)=1 / 3$, then it will immediately follow $p=1$, and the corresponding strong negation $N_{1}(x)=(1-x) /(1+x)$ is yet to be checked before freezing it as the designed functional model for «not».

This paper is mainly devoted to exhibit some plausible arguments in pro of choosing the membership function of a predicate through a careful process of design. As already suggested in the former example, the chosen mathematical frame is fuzzy logic. It is assumed that the readers -"users of the model"- are familiar with its basics.

\section{Some basic concepts}

A predicate is understood in this paper as the name of a property exhibited by the elements of a set $X$. In what follows, $P$ is a predicate, $p$ the property it names, and the meaning of $P$ in $X$ is given just by the use of $P$ in $X$; a use that depends on the purpose for doing it. That is, the meaning of $P$ in $X$ is viewed as something that, arising from some relationships between the elemental statements $« x$ is $P »$, with $x$ in $X$, is established thanks to both the extent up to which each $x$ shows $p$, and its variations among the pairs of elements in $X$.

It will be supposed that the management of $P$ in $X$ is done with the goal of introducing some kind of organization in $X$ trying to clarify how the elements in $X$ become related when $p$ is taken into account. For instance, one thing is just the set of ages of the inhabitants of a big city (a set of numbers that can be taken from one to $33 \times 10^{8}$ seconds), and another and very different thing is that appearing as soon as those inhabitants that are «young», are considered; that is, when the collective of the "young people» in the city is considered. This is the sense in which it can be said that a predicate 'collectivizes', or 'extents', in its universe of discourse, and even if such extension is of a virtual, or mental, character; that is, it has not a physical existence out of the brain. This collective is clearly different from that constituted by the inhabitants that are between 18 and 36 years old, and that, at least ideally, can be made physical by simply placing this people in a list of which the inhabitants with other ages are excluded. On the contrary, and as it can be shown by a Sorites type of argument, not a satisfactory list can be made with the inhabitants qualified by the predicate «young». Most collectives, like that given by «young», are nebulous-like entities that, appearing in the language after using $P$, are very difficult to study in themselves, and, in particular, of being represented in mathematical terms. Nevertheless, collectives do exist in the language. A way of approaching the collectives can be done by functions (fuzzy sets) able to express up to which degree it can be stated that $« x$ is $P »$, for all $x$ in $X$.

\section{Towards describing the 'collective', or 'extent', a predicate generates.}

3.1. To face a scientific-like study of predicates, we will restrict ourselves to those that can be viewed as measurable quantities. To such an end, it will be firstly supposed [6] that the two empirically based binary relations defined in $X$ by,
a) $x={ }_{P} y$ iff $x$ is as equally $P$ as $y$, and
b) $x \leq_{P} y$ iff $x$ is less $P$ than $y .{ }^{1}$

can be (perceptively) captured through the way of using $P$ in $X$, and we will also suppose that the two following working-hypotheses hold:

- $\leq_{P}$ is a preorder (that is, a reflexive and transitive relation), and

- $=_{P}$ is the equivalence associated to $\leq_{P}$ (that is, ${ }_{P}=\leq_{P} \cap \leq_{P}^{-1}$, with $\left.x \leq_{P}^{-1} y \Leftrightarrow y \leq_{P} x\right)$

Notice that only $\leq_{P}$ is strictly necessary, although it is neither obvious that $\leq_{P}$ is always a preorder, nor that $=_{P}$ is always its associated equivalence. Obviously, $=_{P}$ is not the identity: If $x=y$, then $x={ }_{P} y$, but not reciprocally.

In this relational framework, it is supposed that each $x$ in $X$ shows $p$ to some extent, and that $\leq_{P}$ is the organization the use of $P$ induces in $X$. The graph $\left(X, \leq_{P}\right)$, a preordered set, is an interpretation of the elemental, or primary, meaning of $P$ in $X$ that, in the form of the graph, is expressed in mathematical terms allowing to look at it as a, perhaps measurable, qualitative quantity.

If there exists some mapping $m_{P}: X \rightarrow[0,1]$, such that: 1) $x \leq_{P} y \Rightarrow m_{p}(x) \leq m_{P}(y)$, it will be said that $m_{P}(x)$ measures the amount up to which each $x$ is $P$, or exhibits $p$, and that $m_{P}$ is a measure for $P$ in $X$. Additionally, it will be supposed the following two prototype-hypotheses for $m_{P}$ : Provided $\left(X, \leq_{P}\right)$ contains minimals $x_{0}\left(x_{0} \leq_{P} x\right.$, for all $x$ in $X)$, or maximals $x_{1}\left(x \leq_{P} x_{1}\right.$ for all $x$ in $\left.X\right)$, then 2) $m_{P}\left(x_{0}\right)=0$, and 3) $m_{P}\left(x_{1}\right)=1$. Once $\leq_{P}$ and $m_{P}$ are known, it can be said that the triplet $\left(X, \leq_{P}, m_{P}\right)$ is a measurable quantity interpreting the use, or meaning, of $P$ in $X$. Minimals $x_{0}$ play the role of anti-prototypes of $P$ in $X$, and maximals $x_{1}$ play like prototypes of $P$ in $X$ [20]. Obviously, properties 1,2 , and 3 , do not individuate a single measure $m_{P}$, but a family of them; to have a single one, more information on the current behavior of $P$ in $X$ is needed.

1 Stricto sensu it should be " $x \leq_{P} y$ iff $x$ is less $P$ than $y$ or as equally $P$ as $y$ ", however, when no confusion arises, to simplify the linguistic expressions, the shorter form will be preferred. 


\section{Remarks}

1. Property 1 of $m_{P}$ is basic, since it shows that the numbers $m_{P}(x)$ also grow when the elements $x$ 'grow' under $\leq_{P}$.

2. The quotient set $X /=_{P}$ consists of the classes of those elements that are as «equally $P_{\Downarrow}$ as a given one; for instance, and if a minimal $x_{0}$ exists, it is $\left[x_{0}\right]=\left\{x\right.$ in $\left.X ; x={ }_{P} x_{0}\right\}$.

3 . If $x={ }_{P} y$, or $x \leq_{P} y$ and $y \leq_{P} x$, then $m_{P}(x) \leq m_{P}(y)$ and $m_{P}(y) \leq m_{P}(x)$, imply $m_{P}(x)=m_{P}(y)$, that is, all elements in a class $[x]$ have the same measure $m_{P}(x)$; for instance, if $x$ is in $\left[x_{0}\right]$ it is $m_{P}(x)=0$ : Measures $m_{P}$ are constant on the classes in $X /={ }_{P}$.

4. In the quotient set $X /=_{P}$, the relation defined by $[x] \leq^{*}[y] \Leftrightarrow x \leq_{P} y$, does not depend on the chosen representatives $x$ and $y$ in the classes, and provided $\leq_{P}$ is a preorder, then $\leq^{*}$ is a partial order (a reflexive, anti-symmetric, and transitive binary relation). Hence, $\left(X /=_{P}, \leq^{*}\right)$ is a poset, and the mapping defined by $m_{P}^{*}([x])=m_{P}(x)$, is also a $\leq{ }^{*}$-measure.

3.2 Once $\left(X, \leq_{P}\right)$ is endowed with a measure $m_{P}$, the new relation $\leq_{m P}$, defined by

$$
x \leq_{m P} t \Leftrightarrow m_{P}(x) \leq m_{P}(y)
$$

is obviously a linear, or total, preorder that is larger than $\leq_{P}$, since for all $x, y$ it is either $m_{P}(x) \leq$ $m_{P}(y)$, or $m_{P}(x) \geq m_{P}(y)$, and if $x \leq_{P} y$, then $m_{P}(x) \leq m_{P}(y) \Leftrightarrow x \leq_{m P} y: \leq_{P}$ is included in $\leq_{m P}$. Hence, if a working-scientist directly deals with a measure $m_{P}$, he/she is adding to the meaning of $\leq_{P}$ the difference set $\leq_{m P}-\leq_{P}$.

It should be noticed that many times it is difficult to know $\leq_{P}$, especially if the cardinality of $X$ is big; notwithstanding, there are cases in which the universe of discourse can be assimilated to an interval of the real line, and $\leq_{P}$ is either the order $\leq$ in such line, or its reverse $\leq^{-1}$. This is, for instance, the case of $P=$ big in the closed interval $[0,10]$, where it can be easily agreed in the identification $\ll x \leq_{P} y \Leftrightarrow x \leq y »$, with at least the minimal 0 , and the maximal 10 , or the case of $P=$ small with $\ll x \leq_{P} y \Leftrightarrow y \leq x »$, with at least the minimal 10, and the maximal 0 . In the first case, a measure $m_{P}$ should be selected among those that verify $m_{P}(0)=$ $0, m_{P}(10)=1$, and $« x \leq y \Rightarrow m_{P}(x) \leq m_{P}(y) »$, and in the second among those verifying $m_{P}(0)=1$, $m_{P}(10)=0$, and $« x \leq y \Rightarrow m_{P}(y) \leq m_{P}(x) »$. In both cases, and provided $m_{P}$ is strictly nondecreasing (respectively, decreasing), it is $\leq_{P}=$ $\leq_{m P}$. Linear instances of these functions are, respectively, $m_{P}(x)=x / 10$, and $m_{P}(x)=1-x / 10$.

In those cases in which it is $\leq_{P}=\leq_{m P}$, it can be said that $m_{P}$ perfectly reflects the meaning of $P$ in $X$. Nevertheless, since $\leq_{P}$ is not always a total preorder, in general, $m_{P}$ will not perfectly reflect $\leq_{P}$, and then the non-empty set $\leq_{m P}-\leq_{P}$ can produce a modification of the meaning $\leq_{P}$ of $P$ in $X$, by enlarging it to $\leq_{m P}$. The working-scientist should take care of this possibility, and especially, when this enlargement could be excessive for correctly interpreting the meaning of $P$ in $X$ through $m_{P}$.

Example. In $X=[0,10]$, the relation $\leq_{P}$ for the predicate $P=$ «Around Four», can be taken as coincidental with

$$
x \leq_{P} y \Leftrightarrow x \leq y \text {, if both } x \text { and } y \text { are in }[0,4],
$$$$
\text { and } y \leq x \text {, if both are in }[4,10] \text {, }
$$

and showing at least the two minimals 0 and 10, and at least the maximal 4 . Hence, all the measures $m_{P}$ will come from the 'axioms': $m_{P}(0)=m_{P}(10)=0$; $m_{P}(4)=1 ; \ll 0 \leq x \leq y \leq 4 \Rightarrow m_{P}(x) \leq m_{P}(y) »$, and $« 4 \leq x \leq y \leq 10 \Rightarrow m_{P}(x) \geq m_{P}(y) »$. Of course, at each particular case, a single one among this multitude of functions should be selected to represent the current use of the predicate, and thus more information on the particular use of $P$ in $X$ is needed for it. If, for instance, it is known that the numbers in $[0,1] \cup[9,10]$ are minimals, those in $[3.5,4.5]$ are maximals, and that the growing of $m_{P}$ is lineal, then $m_{P}$ will be the function:

$$
\begin{aligned}
& m_{P}(x)=0, \text { if } x \text { is in }[0,1] \cup[9,10] ; \\
& m_{P}(x)=1, \text { if } x \text { is in }[3.5,4.5] ; \\
& m_{P}(x)=(x-1) / 2.5, \text { if } x \text { is in }[1,3.5], \text { and } \\
& m_{P}(x)=(9-x) / 4.5, \text { if } x \text { is in }[4.5,9] .
\end{aligned}
$$

Provided it were known that in the interval $[1,3.5]$ the growing of $m_{P}$ is quadratic, that is, with prototype function $m_{P}(x)=a x^{2}+b x+c$, if $1<x<3.5$, the three parameters can be obtained by simply having an information like $m_{P}(2.5)=0.5$. Then, and jointly with $m_{P}(1)=0$ and $m_{P}(3.5)=1$, it is easy to compute $a, b$, and $c$, and the corresponding measure $m_{P}$ is:

$$
\begin{aligned}
& m_{P}(x)=0, \text { if } x \text { is in }[0,1] \cup[9,10] \\
& m_{P}(x)=1, \text { if } x \text { is in }[3.5,4.5] ; \\
& m_{P}(x)=0.067 x^{2}-4.4 x+4.33, \text { if } x \text { is in }[1,3.5], \text { and } \\
& m_{P}(x)=(9-x) / 4.5, \text { if } x \text { is in }[4.5,9] .
\end{aligned}
$$

\section{Remarks}

1) By considering non numerical measures $m_{P}$ taking their values in a poset $(L, \leq)$ instead of in the unit interval, there is a natural way for obtaining a non numerical measure $\mathbf{m}_{P}: X \rightarrow L$ with which the meaning of $P$ is perfectly reflected [6]. It is enough to take $L=X /=_{P}$, with its partial ordering $\leq^{*}$, and defining $\mathbf{m}_{P}(x)=[x]$. Then, it is: ' $x \leq_{P} y \Leftrightarrow$ $[x] \leq^{*}[y]$, or $\mathbf{m}_{P}(x) \leq^{*} \mathbf{m}_{P}(y) \Leftrightarrow x \leq_{m P}^{*} y^{\prime}$, and $\leq_{P}=\leq_{m P}^{*}$. Hence, $\mathbf{m}_{P}$ perfectly reflects $\leq_{P}$. Thus, in the case of a numerical measure taking its values in $[0,1]$ and perfectly reflecting $\leq_{P}$, it is $\leq_{P}=\leq_{m P}=\leq_{m P}^{*}$, showing the relevance at the 
respect of the equivalence classes $[x]$.

2) With all that, any possible way to descriptively approach the concept of 'collective' cannot forget all the possible measures $m_{P}$. Each one of them will represent a kind of 'state' of the collective, respect to a given scale $(L, \leq)$.

\section{The usual case with Fuzzy Sets}

4.1 Once a triplet $\left(X, \leq_{P}, m_{P}\right)$ is given, the fuzzy set labeled $P(\mathbf{P})$ can be immediately defined through [21]:

- $x$ belongs to $\mathbf{P}$ with degree of membership $r$, $x \in_{r} \mathbf{P}$, if and only if $m_{P}(x)=r \in[0,1]$, with the understanding that $r=0$ means that $x$ does not belong at all to $\mathbf{P}$, and that $r=1$ means that $x$ completely belongs to $\mathbf{P}$.

- Two fuzzy sets $\mathbf{P}$ and $\mathbf{Q}$ are identical, $\mathbf{P}=\mathbf{Q}$, if and only if $m_{P}(x)=m_{Q}(x)$, for all $x$ in $X$, that is, $m_{P}=m_{Q}$.

Notice that each fuzzy set $\mathbf{P}$ is defined by just changing the way of speaking, and that it is completely defined by a function $m_{P}$. That is, provided $m_{P}$ and $m_{P}^{*}$ were two different measures for the same predicate $P$, two different fuzzy sets $\mathbf{P}$ and $\mathbf{P}^{*}$ will be correspondingly defined. In principle, a predicate $P$ allows to define a family of fuzzy sets in $X$ sharing some similar shape. Notice also that a crisp set is a fuzzy set whose measure $m_{P}$ verifies $m_{P}(x) \in\{0,1\}$, for all $x$ in $X$, and that in these cases the extent of $P$ is just the classical subset $m_{P}^{-1}(1)$ in $X$. Classical sets $\mathbf{A}$ are viewed, in this way, as degenerate fuzzy sets for which, in addition, if $\mathbf{A}=\mathbf{B}$, also $\mathbf{B}$ is a classical set. $\mathbf{A}$ 'proper fuzzy set' is one with some value $m_{P}(x)$ in $[0,1]$. In the same vein, no proper fuzzy set can be identical to a crisp set. For crisp sets, the new symbol $\epsilon_{r}$ only admits the two possibilities $\epsilon_{1}(\in)$, and $\epsilon_{0}(\notin)$, and the collective reduces to just the crisp set, something that shows the 'static' character of those predicates that generate crisp sets.

4.2 The membership function of a fuzzy set labeled $P$, is not always obtained by a process like those in the last examples with «big», «small», and «around four», but through one in which only the main features at hand on the behavior of $P$ in $X$, are taken into account. This can be particularly worrying when $X$ cannot be either directly assimilated to a set of real numbers, or the preorder $\leq_{P}$ has nothing to do with the order of the real line. It happens, for instance, with the predicate $F=$ «uzzy», applied to fuzzy sets $\mathbf{A}$, in which case it is accepted that $\mathbf{A} \leq_{s} \mathbf{B} \Leftrightarrow \mathbf{A}(x) \leq \mathbf{B}(x)$, if $\mathbf{B}(x) \leq 0.5$, and $\mathbf{B}(x) \leq \mathbf{A}(x)$, if $0.5 \leq \mathbf{B}(x)$, the so-called 'sharpened order' between fuzzy sets, translates the linguistic relation «A is less fuzzy than $\mathrm{B}$ ». Nevertheless, this definition leaves some doubts in what re- spects to its coincidence with $\leq_{F}$, (less fuzzy than), even if it seems to verify $\leq_{s} \subset \leq_{F}[23]$.

When the preorder $\leq_{P}$ is not well, or fully, known, the membership function $f_{P}$ of a fuzzy set labeled $P$ directly obtained from the information at hand, can easily be not coincidental with one of the measures $m_{P}$, and hence does not define the fuzzy set $\mathbf{P}$ but another one. Let us call $m_{P}$ an 'ideal membership function', and $f_{P}$ a 'practical membership function'; for short, and respectively, an im-function, and a pm-function. Of course, an im and a pm functions are not necessarily identical, even if they should keep some similarity concerning, at least, their shape; for instance, if $m_{P}$ is strictly decreasing in some part of $X$, in this part $f_{P}$ cannot be strictly non-decreasing. Provided, the pm-function $f_{P}$ comes from a good enough information on the behavior of $P$ in $X$, and depending on the characteristics of the current problem, this lack of coincidence could have no importance at all, but in some specific cases as they are, for instance, those in which the values $m_{P}(x)$ are of upmost importance, the numerical differences $\left|m_{P}(x)-f_{P}(x)\right|$ can be of some relevance.

4.3 If, either $\leq_{m_{P}} \subset \leq_{f_{P}}$, or $\leq_{f_{P}} \subset \leq_{m_{P}}$, the 'working meaning' $\leq_{f_{P}}$ could be, respectively, too large, or too short, and since usually it is also $\leq_{P} \subset$ $\leq_{m_{P}}$, by transitivity it will follow $\leq_{P} \subset \leq_{f_{P}}$ in the first case, and $\leq_{f_{P}} \cap \leq_{P} \subset \leq_{m_{P}}$ in the second. Additionally, it should be remarked that $m_{P}$ could perfectly reflect the meaning of $P$, but $f_{P}$ could not. As it is clear, each time the best representation of the meaning of $P$ is at a stake, the pm-function $f_{P}$ plays a role of paramount importance. In fact, and from a qualitative point of view, a function $f_{P}$ can be considered as an approximation to a function $m_{P}$, and the fitness of such approximation can be relevant. At this respect, what can the workingscientist do? The best he/she can do is to design $f_{P}$ in the best possible form, by taking into account as much information as he/she can collect on the behavior of $P$ in $X$. That is, by doing the finest possible design of $f_{P}$.

\section{Remarks}

The example in section 2.2, shows the basic treats for a good enough process of designing the pmfunction of a fuzzy set labeled $P$. The process consists in the following steps:

1. Capture the main aspects on the behavior of $P$ in $X$ (the 'axioms' in the example).

2. Once a shape for a prototype of the membership function is obtained, its final form $f_{P}$ depends on getting more information about either some parameters, or on something else that can come from the particular use of $P$ in $X$. The designer should carefully search for this additional information. 
3. Introducing the additional information into the prototype, the pm-function $f_{P}$ is obtained.

4. Once $f_{P}$ is known, it should be checked against all the known data, to be sure that it satisfies what is required by the current problem.

5. In a positive case, $f_{P}$ is accepted; in a negative, the designer should return to point 1 , and proceed newly through points 2 and 3 .

The process just described is nothing rare in Engineering, where plans of 'design' are a common way of working, for instance to obtain what is necessary with the goal of applying the models with which computations can be made.

\section{Beyond predicates}

The former sections presented a comprehensive view of modeling, designing, possibly imprecise predicates with very simple mathematical structures: partial ordered sets, equivalence relations and the resulting quotient sets, partitions and classes. The next level of complexity would be the modeling of linguistic variables [24], whose "values" are linguistic terms, i.e., predicates. In this case, the additional design aspects include the definition of a universe of discourse -the domain of the linguistic variable- and its granularity- the number and distribution of the linguistic terms in the universe. The psychology rule "seven plus minus two" [10] helps for a preliminary choice of the number of linguistic terms, which should be complemented with additional knowledge about the linguistic variable under modeling to complete the design. Predicates and linguistic variables represent linguistic statements. Possibly the simplest, but most used, models of systems associated to fuzzy logic are the rule-based ones:

«if conditions(s) then conclusion»,

where the «if conditions(s)» part implies that if the stated conditions are satisfied, then a conclusion, possibly constrained by the degree of satisfaction of the conditions, will follow. The «if conditions(s)» part has the structure «if $x_{1}$ is $X_{1}$ and ... and $x_{n}$ is $X_{n}$ », where $x_{i}, X_{i}(i=1,2, \ldots, n)$ represent a physical and a linguistic term of an associated linguistic variable, respectively, and "is" formally denotes measuring the similarity between a singleton with the actual value of $x_{i}$ and the fuzzy set representing $X_{i}$. To model this part of a rule, the connective "and" has to be considered. Notice that when a human being is using a rule of this kind, with "and", s/he is meaning that the conditions should be satisfied at the same time. In the case of fuzzy logic, "and" is meant to denote an aggregation of the degrees of satisfaction of the conditions. For this, mostly t-norms [2], [3], [4] are used. (There are however systems -compensating systems- which cannot be properly modelled with t-norms, and other aggregations, (frequently linear or non-linear combi- nations of t-norms and t-conorms) are required [25], [13]). The problem for the model builder is, that there exist infinite many t-norms. It is obviously a very hard, and still an open problem, how to find the right one. There are however serious studies on the consequences of a bad choice (see e.g. [19], [22]). Before discussing how to solve this problem, (see below), some remarks about the «conclusion» part are needed. Conclusions may be of two general types: crisp actions, («if the book is not expensive then buy it»), or fuzzy statements («if the external temperature is low then the heating demand will be high»). As in the former case, besides modeling the statements, the main problem here is the appropriate choice, among many, of the conditional to realize the connecting "then".

\section{Data driven modeling}

Particularly in our days it is not rare to have a good amount of behaviour data of the systems we are faced to model. In this case it is possible to take advantage of the capabilities of other members of the Soft Computing consortium to realize "data driven fuzzy modeling", instead of making a blind choice of operations and prototypical shapes of fuzzy sets. For instance, the learning capability of neural networks can be used to "learn" membership functions of the conditions-fuzzy sets [9], [1], [5], [11], [17] or to learn aggregation connectives [12], to use data driven evolutionary algorithms to optimize the distribution of linguistic terms in a given universe of discourse or even to optimize full fuzzy rules sets [7], to adjust the shape of the transitions between cosupport and core of fuzzy sets [15], or to design and adjust parameterized conjunctions and conditional operations [14], [16].

\section{Conclusions}

This paper is mainly devoted to present some plausible reasons in pro of carefully designing the membership function of fuzzy sets, based on the modification of the meaning that $P$ can show with just a rough and quick design of the membership function. Nevertheless, it should be noticed that when representing in fuzzy terms a dynamical system whose behavior is known through a linguistic description of it, all the predicates, linguistic connectives and modifiers, quantifiers, conditional statements, etc., should also be carefully designed in mathematical terms. (See the chapter Conclusions of [18]).

A good help for the designer of a fuzzy system could come from some of the papers of a mathematical character that, usually published under the rubric 'fuzzy something', contain abstract models potentially applicable to the problems in the applications. This, of course, provided those models can be designed to fit well enough with the necessary requisites in the corresponding application [22], 
[16].

With all that, and just for a didactical purpose, it seems recommendable to improve the famous Zadeh's statement,

\section{In fuzzy logic everything is a matter of degree,}

by completing it to the form,

\section{In fuzzy logic everything is not only a matter of degree, but also of design.}

Hence, engineers needing to become fuzzypractitioners, up to some extent should master the 'art of designing fuzzy systems'. This art can suggest some new questions like, for instance:

Why, in fuzzy ruled systems, and as it is currently done in Fuzzy Control, all the rules are always represented by the same conditional, or implication function, but not each rule by a different one?

Preliminary positive results on a fuzzy rule-based regression system with diversity of operations for the rules may be found in [16]. It is beyond discussion that the same question is important if the specification of the system or some of its relevant signals are expressed in natural language, i.e., if we enter the world of "Computing with Words".

\section{References}

[1] H.G. Allende-Cid, A. Veloz, R. Salas, S. Chabert, H.M. Allende: Self-organizing neurofuzzy inference system, LNCS 5197, 422- 429, 2008

[2] C. Alsina, E. Trillas, Ll. Valverde: Sobre conectivos lógicos no-distributivos para la teoría de conjuntos borrosos, (in Spanish). Pubs. Matemáticas de la Universidad Autónoma de Barcelona, 20, 69-72, 1980

[3] C. Alsina, E. Trillas, Ll. Valverde: On nondistributive logical connectives for fuzzy sets theory, BUSEFAL 3, 18-29, 1980

[4] C. Alsina, E. Trillas, Ll. Valverde: On some logical connectives for fuzzy sets theory. Jr. Math. Analysis Appl. 93, 15-26, 1983

[5] J.M. Benítez, J.L. Castro, I. Requena: Are neural networks black boxes? IEEE Trans. on Neural Networks 8, 1156-1163, 1997

[6] I. García-Honrado, E. Trillas: An essay on the linguistic roots of fuzzy sets. Information Sciences 181, 4061-4074, 2011

[7] F. Herrera, J.L. Verdegay (Eds.): "Genetic Algorithms and Soft Computing". Physica Verlag, Heidelberg, 1996

[8] Internet: http://interstitiality.net/BD/ brainEngDiagram.html

[9] Jang J-S. R.: ANFIS: Adaptive-network-based fuzzy inference system, IEEE, Trans. Systems, Man and Cybernetics 23 (3), 665-685. 1993
[10] G.A. Miller: The magical number seven, plus or minus two. Some limits on our capacity for processing information. Psychological Review, 101 (2), 343-352, 1994

[11] C. Moraga: Neuro-fuzzy modeling of compensating systems. In: "Quo vadis Computational Intelligence?", (P. Sinčák, J. Vaščák, Eds.), 385398. Physica-Verlag, Heidelberg, 2000

[12] C. Moraga: Neuro-evolutionary systems for learning parametric fuzzy connectives from examples of behaviour. Proc. Workshop on Data Mining and Automatic Learning. Conference IBERAMIA 2002, Sevilla, Spain, 2002

[13] C. Moraga: Compensating systems: a challenge for neuro-fuzzy modeling. Int. Journal of Systemics, Cybernetics and Informatics 1 (1), 16-20. ISSN 0973-4864, 2006

[14] C. Moraga, A. Pradera, E. Trillas: Evolutionary tuning of fuzzy if-then rules at the level of operations: A proposal. Actas II Congreso Español MAEB, 530-537. Ed. Universidad Oviedo. ISBN 84-607-65-26-1, 2003

[15] C. Moraga, R. Salas: A new aspect for the optimization of fuzzy if-then rules. Proc. 35th IEEE Inter. Symposium on Multiple-valued Logic, 160165, IEEE-CS-Press, 2005

[16] C. Moraga, M. Sugeno, E. Trillas: Optimization of fuzzy if-then rule bases by evolutionary tuning of the operations. 221-226. Proc. 39th Int. IEEE Symposium on Multiple-valued Logic. IEEE-CS-Press, 2009

[17] C. Moraga, K.-H. Temme: S-neural networks are fuzzy systems. Proc. Int. Conference on Information Processing and Management of Uncertainty. Madrid, Spain, 1518-1523, 2000

[18] E. Trillas: "Conjuntos Borrosos", (in Spanish). Editorial Vicens-Vives, Barcelona, 1980

[19] E. Trillas: Lógica borrosa y narrativa: un párrafo de Vila-Matas. Proc. XII Congreso Español Tecnologías y Lógica Fuzzy, 15-20, (in Spanish). ISBN 84-609-2160-3, 2004

[20] E. Trillas: On the genesis of fuzzy sets. Agora 27, (1), 7-33, 2008

[21] E. Trillas: On the use of words and fuzzy sets. Information Sciences 176, (11), 1463-1487, 2006

[22] E. Trillas, S. Guadarrama: Fuzzy representations need a careful design. Int. Jr. General Systems, Vol. 39 (3), 329-346, 2010

[23] E. Trillas, D. Sánchez: A briefing on fuzziness and its measuring. Proc. Int. Conference on Information Processing and Management of Uncertainty, 15-24, 2012

[24] L.A. Zadeh: The concept of a Linguistic Variable and its application to approximate reasoning. Information Sciences 1, (8), 199-249; 2, (8), 301-357; 3, (9), 43-80, 1975

[25] H.-J. Zimmermann, P. Zysno: Decisions and evaluations by hierarchical aggregation of information, Fuzzy Sets and Systems 10, 243-266, 1983 